\title{
Dynamics of $\gamma$-tubulin cytoskeleton in HL-60 leukemia cells undergoing differentiation and apoptosis by all-trans retinoic acid
}

\author{
AHMAD SHARIFTABRIZI $^{1,2}$, SHAHIN AHMADIAN ${ }^{2}$ and YAGHUB PAZHANG $^{3}$ \\ ${ }^{1}$ Department of Clinical Biochemistry, Tehran University of Medical Sciences; ${ }^{2}$ Department of Biochemistry, \\ Institute of Biochemistry and Biophysics, University of Tehran, Tehran; ${ }^{3}$ Islamic Azad University, Urmia Branch, Urmia, Iran
}

Received June 24, 2011; Accepted August 17, 2011

DOI: $10.3892 / \mathrm{mmr} .2011 .635$

\begin{abstract}
Microtubules are important components of the cell cytoskeleton, participating in protein localization and cell signaling. The capacity of leukemia cells to re-organize their microtubules is considered an integral part of differentiation in these cells in order to become mature granulocytes through treatment with all-trans retinoic acid (ATRA), an established drug for treating acute promyelocytic leukemia. In this study we examined $\gamma$-, $\alpha$ - and acetylated- $\alpha$-tubulin content, their patterns of distribution in the cytoplasm, and the potency of centrosomes in re-organizing microtubules in different stages of ATRAinduced differentiation and apoptosis of the HL-60 cell line. The $\gamma$-tubulin content was dramatically increased following differentiation of HL-60 cells, and was then decreased after apoptosis. We also found that $\gamma$-tubulin had a diffuse, cytoplasmic pattern following apoptosis compared to the focal, centrosomal accumulation of $\gamma$-tubulin in differentiated cells. Differentiated cells had the ability to re-organize their microtubule network following nocodazole challenge testing, whereas undifferentiated cells did not show a similar ability. $\alpha$-tubulin was more regularly organized in differentiated cells, and did not reveal any specific pattern of polymerization in apoptotic cells. Acetylated- $\alpha-$ tubulin generally followed the same organization patterns after differentiation, as that which occurred for $\alpha$-tubulin. Our data is suggestive of a centrosomal and organized nucleation pattern of microtubules in HL-60 cells following differentiation, possibly mediated through up-regulation of $\gamma$-tubulin.
\end{abstract}

\section{Introduction}

Differentiation therapy of cancer cells involves induction of differentiation in these cells, thus preventing further prolifera-

Correspondence to: Dr Shalin Ahmadian, Institute of Biochemistry and Biophysics, University of Tehran, Tehran 14114, Iran

E-mail: ahmadian@ibb.ut.ac.ir

Key words: apoptosis, differentiation, $\gamma$-tubulin, HL-60 cells, microtubules tion. Clinically, the most successful example of differentiation therapy is treatment of acute promyelocytic leukemia with all-trans retinoic acid (ATRA), as it causes very high rates of remission in patients by inducing differentiation and apoptosis of promyelocytes. Central to the pathogenesis of acute promyelocytic leukemia (APL) is expansion of malignant myeloid cells blocked at the promyelocytic stage of differentiation, along with reciprocal chromosomal translocations involving the retinoic acid receptor $(R A R \alpha)$ gene on chromosome $17 \mathrm{q} 21$. ATRA may induce myeloid differentiation by up-regulating the RAR/RXR complexes, thus overcoming the dominant negative PML-RAR $\alpha$ in APL cells $(1,2)$. The HL-60 cell line serves as a standard in vitro model of differentiation therapy for leukemia with ATRA and is shown to undergo differentiation with ATRA via the RAR/RXR heterodimer (3).

During the processes of differentiation and apoptosis, the cytoskeleton undergoes several critical changes. The main components of the cytoskeleton, specifically the microtubules, microfilaments and intermediate filaments, form a structural network that connects cellular membranes, intracellular organelles and the nucleus. Cellular differentiation involves spatial organization, which is created and maintained by modulation of dynamic instability of microtubules; tight control of which might be of prime importance in the arrangement and orientation of microtubule network during cellular differentiation (4-7). Animal cells in the inter-phase contain a cytoplasmic array of microtubules radiating out, usually from a single microtubule organizing center (MTOC) or centrosome, located near the nucleus, with the minus ends (-) embedded in the centrosome, and the plus ends (+) projecting outward toward the cell surface. MTOC represents the specific location from which microtubules are nucleated. A major component of the MTOC is $\gamma$-tubulin, which shows a $28-35 \%$ homology with $\alpha$ - and $\beta$-tubulin and is expressed in lower amounts than these latter isoforms. Anti- $\gamma$-tubulin antibodies inhibit microtubule re-growth from the centrosome in permeabilized mammalian cells, suggesting that microtubule nucleation depends essentially on $\gamma$-tubulin throughout the cell cycle $(8,9)$. In association with other components, $\gamma$-tubulin forms a 25-nm diameter ring complex for microtubule nucleation (10). $\gamma$-tubulin is also present in the cytoplasm as a smaller complex (11), which constitutes exchangeable stock of matrix 
(12). This process of microtubule nucleation can be achieved through the function of cytosolic or centrosomal $\gamma$-tubulin (13). Non-centrosomal MTOCs, which also contain $\gamma$-tubulin, are shown to play essential roles in plants, as well as in the developing murine embryo $(14,15)$. In the present study, we analyzed changes in microtubule cytoskeleton during the differentiation and apoptosis processes, with an emphasis on the role of $\gamma$-tubulin.

\section{Materials and methods}

Cell culture and induction of differentiation. An established HL-60 human myeloid leukemia cell line (Iranian National Cell Bank, NCBI code C427) was chosen for the experiments. Cells were maintained in RPMI-media (Gibco Inc.) containing L-glutamine and HEPES, supplemented with $10 \%$ fetal calf serum and antibiotics: $100 \mathrm{U} / \mathrm{ml}$ of penicillin and $100 \mu \mathrm{g} / \mathrm{ml}$ of streptomycin. Cell culture was carried out under standard conditions: at $37^{\circ} \mathrm{C}$ in an atmosphere of $95 \%$ air, $5 \% \mathrm{CO}_{2}$. All-trans retinoic acid (ATRA) (Sigma Chemical Co., St. Louis, MO, USA) was used to induce apoptosis and the accompanying differentiation into the granulocyte stage. At the beginning of each experiment, $5 \mathrm{ml}$ of cell suspension was seeded at a concentration of $10^{6}$ cells $/ \mathrm{ml}$ of complete RPMI, into $25-\mathrm{cm}^{2}$ cell culture flasks. Immediately after inoculation, a solution of ATRA was added to achieve a final concentration of $10^{-6} \mathrm{M}$. During the differentiation process, the medium was removed at 48-h intervals and replaced with fresh medium containing ATRA. Simultaneously, fresh medium free of ATRA was also changed in the control flasks. For an assessment of cytoskeleton morphology in each experiment, paired sets of both experimental samples and controls were evaluated.

Immunofluorescence microscopy. Cell solution was first centrifuged at low speed ( $350 \mathrm{x} \mathrm{g}$ ) on poly-L-lysine coated coverslips placed at the bottom of a 50-ml Falcon tube, and the coverslips bearing cells were then immersed in $-20^{\circ} \mathrm{C}$ methanol for $10 \mathrm{~min}$. The coverslips were then rehydrated with PBS, and were washed three times with Ab buffer (PBS, $3 \%$ BSA, $0.1 \%$ Triton X-100, 0.02\% azide). Primary antibodies (anti- $\alpha$-tubulin, cat. no. T9026; acetylated- $\alpha$-tubulin, cat. no. T-6793; and $\gamma$-tubulin, cat. no. T-6557; all from Sigma) were diluted in Ab buffer (all at a 1/100 final concentration) and added to the coverslips which were then incubated for $90 \mathrm{~min}$ at room temperature. Samples were washed three times with $\mathrm{Ab}$ buffer and incubated in a secondary fluorescent-conjugated antibody (FITC-conjugated rabbit anti-mouse antibody, cat. no. F-2012; Sigma) for $45 \mathrm{~min}$. Cells were washed once in the $\mathrm{Ab}$ buffer, and were then incubated in Hoechst 333492 (Sigma cat. no. B2261) at a concentration of $1 \mu \mathrm{g} / \mathrm{ml}$ for $30 \mathrm{~min}$ for DNA staining. Cells were washed once, and coverslips were mounted on slides using a glycerol-containing mounting buffer [1 volume 0.2 $\mathrm{M} \mathrm{Na}_{2} \mathrm{HPO}_{4}(\mathrm{pH} 9.0), 9$ volumes of glycerol]. Samples were observed under a Zeiss fluorescence microscope, and fluorescence micrographs were recorded using a CCD camera. Cells were visualized with DAPI (Ex, $330 \mathrm{~A}$; emission, 480) and FITC filters.

Nocodazole assay. Nocodazole was dissolved in dimethylsulfoxide and was added to the cells at a final concentration of $10 \mathrm{ng} / \mathrm{ml}$. Cells were incubated for $30 \mathrm{~min}$ with medium containing nocodazole and $10 \%$ FCS. Cells were then washed twice with PBS and were incubated for $2 \mathrm{~h}$ in a nocodazolefree medium. Cells were then harvested and subsequently processed for immunofluorescence staining for $\alpha$-tubulin as previously described.

Immunoblot analysis. An equal number of cells were lysed using three cycles of $30 \mathrm{sec}$ sonication on ice in lysis buffer (1 mM DTT, 1 M EDTA, $10 \mu \mathrm{M}$ PMSF, $2 \mathrm{M}$ sucrose) and were then centrifuged for $45 \mathrm{~min}$ at $2,000 \mathrm{rpm}$ at $4^{\circ} \mathrm{C}$. The protein concentration in the lysate was measured using the Bradford assay. Equal amounts of proteins $(10 \mu \mathrm{g})$ were mixed at a $1: 1$ ratio using a sample buffer ( $1 \%$ SDS, $2.5 \mathrm{ml}$ mercaptoethanol, $5 \mathrm{cc}$ glycerol, $1 \mathrm{mg}$ bromophenol blue, $1.78 \mathrm{~g}$ Tris), loaded into mini Lamelli acrylamide gels (5\% stacking gel, $15 \%$ resolving gel) and electrophoresed at a constant voltage of $100 \mathrm{~V}$ for $3 \mathrm{~h}$, and then transferred overnight to nitrocellulose membranes at a 60-mA constant current [utilizing one volume of transfer buffer: $390 \mathrm{mM}$ glycine, $480 \mathrm{mM}$ Tris-base (pH 8.3), 2 volumes of methanol, 7 volumes of distilled water]. Membranes were incubated for $1.5 \mathrm{~h}$ in the blocking solution containing $10 \%$ skim milk in phosphate-buffered saline (PBS) and then incubated in the primary antibodies for $2 \mathrm{~h}$. All of the primary antibodies were diluted in dilution buffer [PBS (pH 7.4), $0.05 \%$ Tween-20, 1\% BSA]. Primary antibodies were against $\alpha$-tubulin (final dilution $1 / 5000$ ), acetylated- $\alpha$-tubulin $(1 / 5000)$ and $\gamma$-tubulin (1/5000).

Membranes were washed 3 times for $5 \mathrm{~min}$ in washing buffer [PBS (pH 7.5), 0.5\% Tween-20] and incubated with secondary antibodies for $1 \mathrm{~h}$ [ALK-P conjugated for $\gamma$-tubulin detection (Sigma cat. no. A1293), detected using BCI/PNBT substrate (Sigma cat. no. B-5655), D=1/20000; and HRP-conjugated for $\alpha$ and $\alpha$-acetylated; Sigma cat. no. A-9044, D=1/1000, detected using DAB substrate (Sigma cat. no. D-8001]. Membranes were incubated in the corresponding substrates for secondary antibodies after a triple wash until the bands were developed and these were then air-dried.

Flow cytometry for cell surface antigens. Cells were incubated in the CD11b FITC-conjugated solution (Dako) for $30 \mathrm{~min}$, washed with PBS three times and analyzed with the BectonDickinson flow cytometer.

NBT assay. Nitro-blue tetrazolium (NBT) assay was carried out using a premixed solution of NBT [4-nitroblue tetrazolium (2 mg), phorbol 12-myrisate 13 -acetate] (200 ng/ml distilled water). Equal volumes of NBT solution and cell solution were mixed and incubated for $30 \mathrm{~min}$ at $37^{\circ} \mathrm{C}$. Cells were then mounted on a slide. Differentiated cells acquired a dark brown color (upon formation of formazan dye from nitroblue tetrazolium after initiation of hexose monophosphate shunt).

DNA laddering. Cells were lysed with digestion buffer $(10 \mathrm{mM}$ Tris- $\mathrm{HCl}, 100 \mathrm{mM} \mathrm{NaCl}, 25 \mathrm{mM}$ EDTA, 1\% SDS, $0.2 \mathrm{mg} / \mathrm{ml}$ proteinase $\mathrm{K}$ ) and were incubated at $56^{\circ} \mathrm{C}$ for $2 \mathrm{~h}$. Cells were subsequently incubated at $37^{\circ} \mathrm{C}$ after addition of Ribonuclease A $(0.2 \mathrm{mg} / \mathrm{ml})$. The lysate was extracted twice with phenol/ chloroform/isoamyl alcohol (25/24/1), and once with chloroform/isoamyl alcohol (24/1). DNA was precipitated by adding 
ammonium acetate to a final concentration of $2.5 \mathrm{M}$, mixed and 2 volumes of pure ethanol was added and then incubated at $-20^{\circ} \mathrm{C}$ overnight. DNA was pelleted by centrifugation at $12,000 \mathrm{x} g$ for $30 \mathrm{~min}$, washed with $70 \%$ ethanol, air-dried and dissolved in the TE buffer $[10 \mathrm{mM}$ Tris- $\mathrm{HCl}(\mathrm{pH} 8.0)$, $1 \mathrm{mM}$ EDTA] and visualized using ethidium bromide-stained agarose gel.

Annexin $V$ assay. Annexin $\mathrm{V}$ assay was performed using a commercial kit (IQ Products, The Netherlands). Briefly, cells were washed twice in calcium buffer, incubated for $20 \mathrm{~min}$ at $4^{\circ} \mathrm{C}$ in the dark in Annexin V-FITC, washed with calcium buffer and incubated for $10 \mathrm{~min}$ with propidium iodide (PI) and analyzed using flow cytometry.

\section{Results and Discussion}

ATRA-induced biochemical and morphological changes in HL-60 cells. Cultured HL-60 cells, in the absence of a differentiation agent, are predominantly promyelocytes, with $<5 \%$ of the cells becoming mature granulocytes (band and segmented neutrophils). ATRA induced changes in the morphology of HL-60 cells characteristic of terminal differentiation in myeloid cells. After 5 days of incubation in $1 \mu \mathrm{M}$ ATRA, over $80 \%$ of HL-60 cells had the phenotype associated with mature granulocytes. Inhibition of growth was observed 3 days after the ATRA exposure compared to the untreated control, but a significant inhibition of growth occurred 4-5 days after treatment with ATRA (Fig. 1A). The ability to reduce NBT and the expression of the mature granulocyte surface marker CD11b were used as differentiation markers of HL-60 cells. No NBT or CD11b positivity occurred during the initial 2 days after exposure to ATRA. However, NBT positivity (Fig. 1B) and CD11b positivity (Fig. 1C) were observed after 3 days, and continued to increase through 5 days after treatment with ATRA. After $96 \mathrm{~h}$ of treatment, the presence of apoptotic cells in a mixed population of cells was also confirmed both by DNA laddering (Fig. 2A) and Annexin V staining (Fig. 2B). The percentage of necrotic cells, as determined by PI staining, was not different in the ATRA-treated and control cells, implying that observed changes were mostly attributable to the apoptotic process. Individual cells were determined to be differentiated, apoptotic or non-differentiated on the basis of characteristic changes in nuclear morphology as assessed by Hoechst staining. Cells with multi-lobulated nuclei were considered differentiated HL-60 cells, and apoptosis was characterized by fragmented nuclei $(16,17)$.

The $\alpha$-tubulin structure and content undergo specific changes following differentiation and apoptosis. The tubulin cytoskeleton has previously been shown to undergo specific changes following ATRA treatment of leukemia cells. As seen by electron microscopy in HL-60 cells, undifferentiated cells possess very few microtubules around the centrosome, but in differentiated cells, numerous microtubules radiating from the centrosome and ending at the cell surface are observed (18). Olins et al, using confocal microscopy in HL-60 cells, showed that centrosomal regions appear more juxtanuclear in undifferentiated cells, yet they migrate to a more distal location following differentiation. Microtubules have an ovoid
A

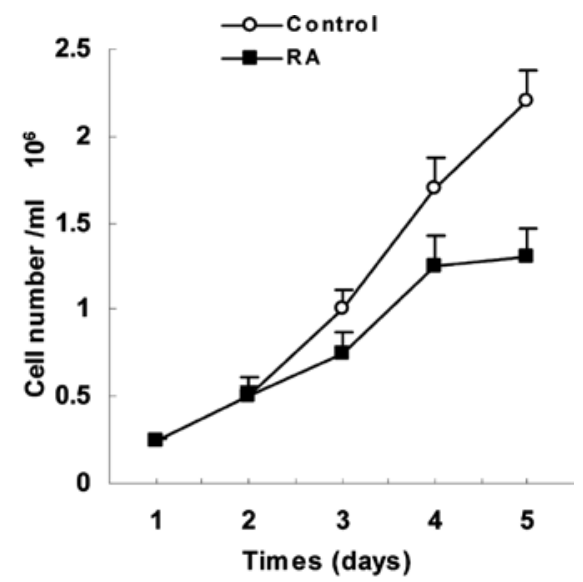

B

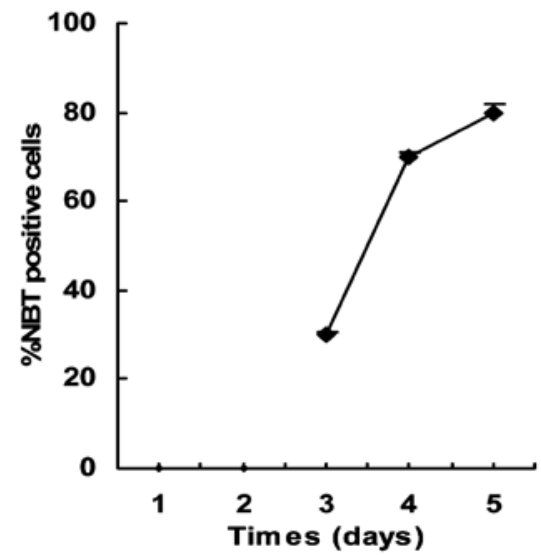

C

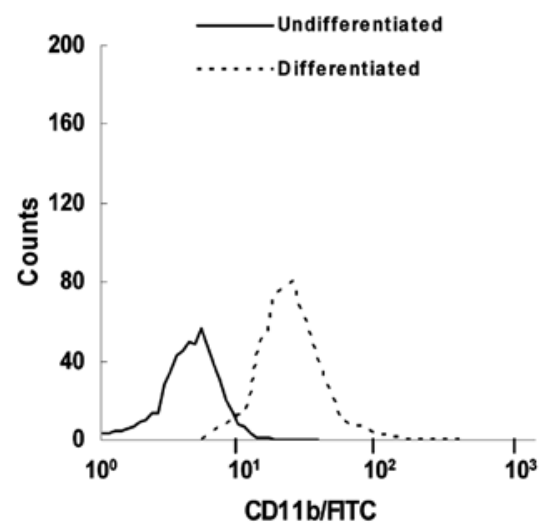

Figure 1. Effect of ATRA on the proliferation and differentiation of HL-60 cells. Cells were treated with $1 \mu \mathrm{M}$ ATRA and, at the indicated times, cell number (A) and NBT positivity (B) were determined. Values represent the means of three separate experiments (Standard Error is represented by bars). Flow cytometry analysis was used to determine the expression of CD11b in the undifferentiated and differentiated HL-60 cells (at day 4) in response to treatment with ATRA (C).

configuration surrounding the nucleus, but in differentiated cells they acquire a shape of parallel array, emanating from the centrosomal origin (19). We observed this same pattern of change in the $\alpha$-tubulin network following ATRA differentiation of HL-60 cells by immunofluorescent microscopy (Fig. 3). In TPA-induced differentiation of HL-60 cells, macrophage differentiation is induced and a random pattern of microtubules are altered to reveal a regular network of arrays, originating from around the nucleus and terminating at the cell 
A

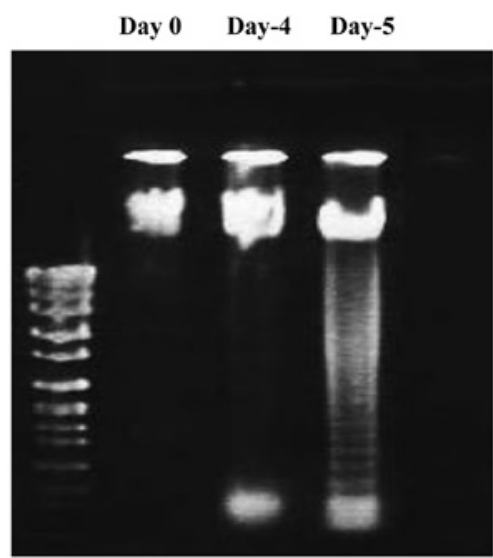

B

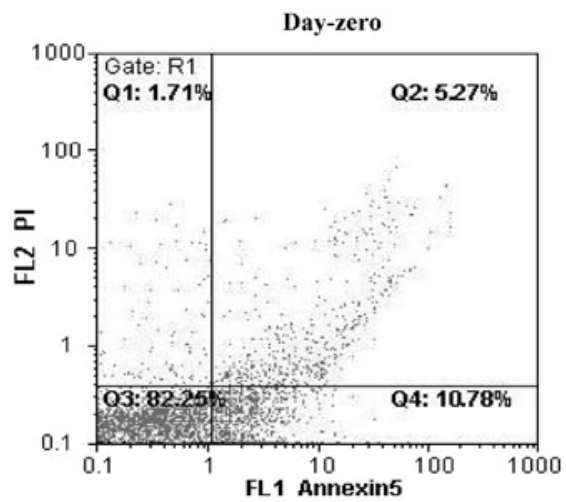

96-hours following ATRA treatment

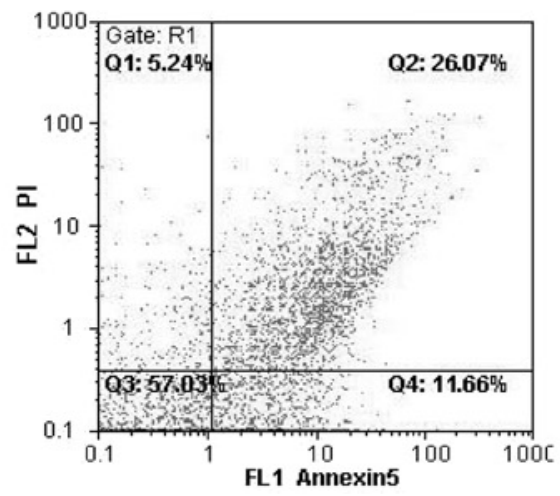

Figure 2. Detection of apoptosis. Apoptosis was documented after 4 days of ATRA treatment by DNA laddering (A). Indications for apoptosis were also analyzed by Annexin V assay. The percentage of cells positive for Annexin V staining was significantly increased after four days of ATRA treatment (B).

periphery (21). Previous studies have reported contradictory results concerning the content of $\alpha$-tubulin after ATRAdifferentiation in HL-60 cells. In one such study, increased $\alpha$-tubulin content was shown as assessed by flow cytometry (20). However, the $\alpha$-tubulin content has also been shown to be stable following ATRA-induced differentiation (19) using Western blotting. We obtained different results by Western blotting, showing a slight reduction in the $\alpha$-tubulin content (Fig. 4A). This could possibly be due to a variation in the methods of detection and/or sensitivities of the various antibodies utilized. Nocodazole treatment has been shown to ablate nuclear lobulation by ATRA, suggesting that microtubules are possibly implicated in the nuclear lobulation process (22). This

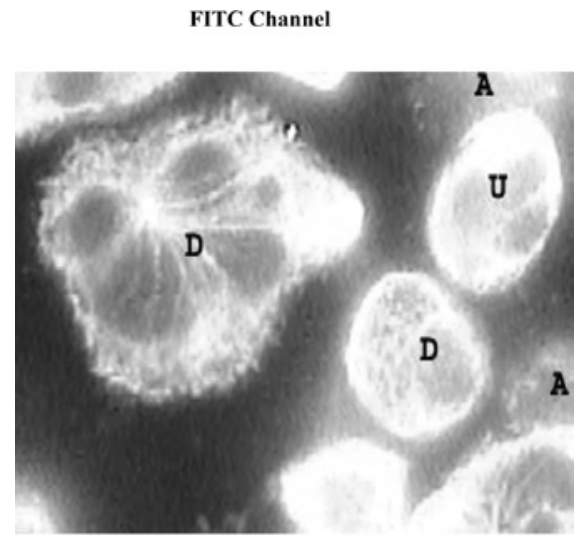

DAPI Channel

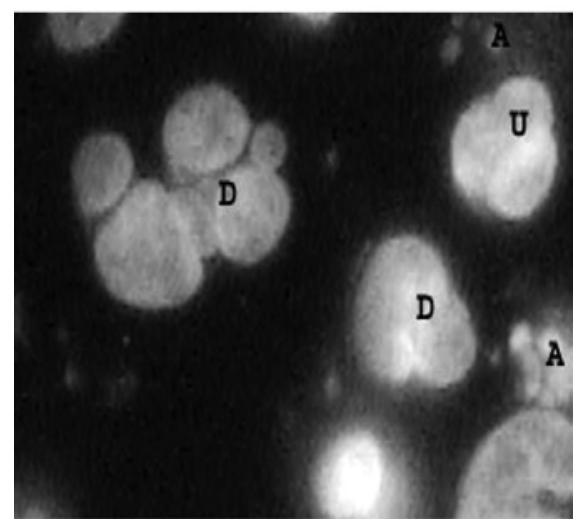

Figure 3. Immunofluorescence staining of $\alpha$-tubulin in a mixed population of HL-60 cells. Differentiated cells showed a radiating array of microtubules originating from the centrosome, while apoptotic cells showed a faint pattern of disperse staining. Undifferentiated cells generally showed a perinuclear pattern of staining (FITC channel). Nuclear morphology was determined by Hoechst staining in the same cells (DAPI channel). U, undifferentiated; D, differentiated; A, apoptotic.

view has been critiqued by a more recent report (23), using a different sub-line of HL-60 cells. Functionally, assembly of microtubules is reported to be crucial for the phagocytosis of differentiated monocytic HL-60 cells (24). We also observed a decreased amount of $\alpha$-tubulin following apoptosis compared to differentiated cells (Fig. 4A). This is comparable to findings in a previous report showing microtubule disruption in ATRAinduced apoptosis of HL-60 cells (21). Cytotostatic drugs, such as doxorubicin, would also lead to microtubule disruption and formation of blebs positively staining for tubulin in HL-60 cells after apoptosis (25). Nevertheless, it is not inevitably predictable that the content of tubulin would be decreased in all apoptotic cells (26).

Content of $\gamma$-tubulin, cytoplasm distribution of $\gamma$-tubulin and microtubule nucleation capacity of centrosomes are dramatically altered in differentiated and apoptotic cells. $\gamma$-tubulin staining was more intense in the differentiated HL-60 cells in the presumptive centrosomal areas than in the non-ATRA-treated cells. Cytoplasm was also diffusely stained. In normal (undifferentiated) cells, a faint localized and very weak cytoplasmic staining for $\gamma$-tubulin was observed. In apoptotic cells, there was no centrosomal staining, but areas of cytoplasmic staining were 

$\mathbf{U}$ D $\mathbf{D}+\mathbf{A}$

B

$\mathbf{U}$

D

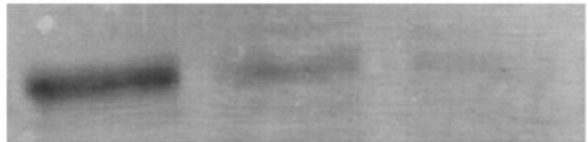

C

$\mathbf{U}$

D

$\mathbf{D}+\mathbf{A}$

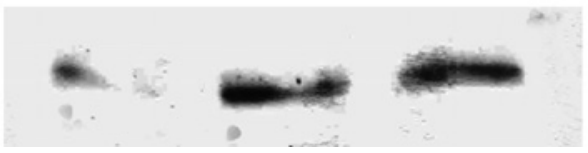

Figure 4. Immunoblot analysis. The cellular content of $\alpha$-tubulin (A), acetylated- $\alpha$-tubulin (B) and $\gamma$-tubulin (C) in undifferentiated (U), differentiated (D) and mixed (differentiated plus apoptotic) (D+A) population of HL-60 cells was determined.

more intense than both differentiated and undifferentiated cells (Fig. 5).

Normal and ATRA-treated cells were incubated with nocodazole. Upon removal of nocodazole, the recovery of the microtubule network was observed at various time intervals. No nucleation was observed after $2 \mathrm{~h}$ of recovery in the undifferentiated cells following nocodazole treatment. There was a disperse staining for microtubules in these cells, consistent with a depolymerized microtubule network. Nearly complete reversal of the microtubule network in the differentiated cells was observed after $2 \mathrm{~h}$ (Fig. 6).

Thus, based on our results, the $\gamma$-tubulin content was increased in the differentiated cells (Fig. 4) and they were localized mostly to centrosomal areas. Centrosomes were observed to be more potent in nucleating microtubules after differentiation, as evidenced by the nocodazole assay. Cumulatively, these data may be considered as evidence of the role of $\gamma$-tubulin in nucleating microtubules in ATRA-induced differentiation of HL- 60 cells. Expression of the CD11b cell surface antigen is an integral part of HL-60 cell differentiation (27). Microtubule re-organization is shown to be a crucial event in the transport of CD11b-containing vesicles in TPA-induced differentiation of U937 cells. U937 cells form the basis of an acute promyleocytic leukemia cell line (28). Our data imply that the process of microtubule re-organization is equally as important in HL-60 cell differentiation and that $\gamma$-tubulin is most likely a key element in this process.

Tubulin self-assembles in vitro when a critical concentration is exceeded, indicating that MTOCs are not absolutely required for microtubule nucleation. However, even in the normal cytoplasm of living cells, the concentration of tubulin is not high enough to account for a free nucleation of microtubules, independent of an MTOC (29). Yet, overexpression of $\gamma$-tubulin is shown to be associated with ectopic microtubule
FITC Channel

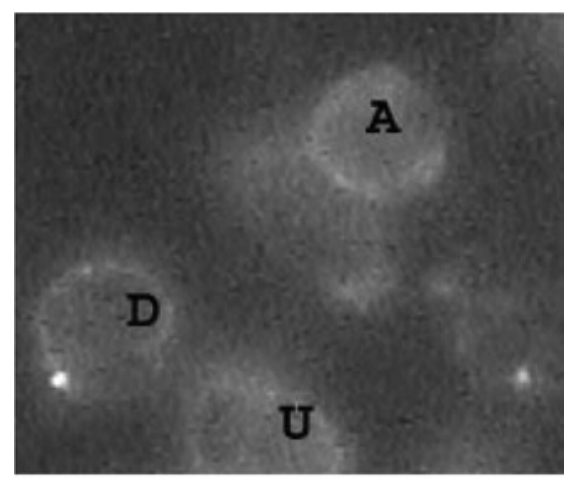

DAPI Channel

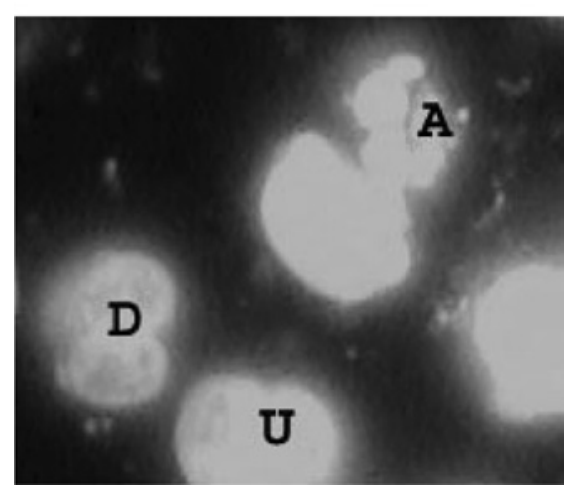

Figure 5. Immunofluorescence staining of $\gamma$-tubulin in a mixed population of HL-60 cells. $\gamma$-tubulin staining was more intense and was mostly observed as juxtanuclear aggregates in differentiated HL-60 cells. A faint staining for $\gamma$-tubulin was observed in undifferentiated and apoptotic cells (FITC channel). Nuclear morphology was determined by Hoechst staining in the same cells (DAPI channel). U, undifferentiated; D, differentiated; A, apoptotic.

nucleation outside the centrosome in mammalian cell cytoplasms (30). The increased cytoplasmic staining of $\gamma$-tubulin, following apoptosis, as observed in our study, may be supportive of this hypothesis, although we did not show directly that this staining would correspond to MTOCs. Induction of apoptosis by the cytostatic drug Taxol in HL-60 cells (25) and for VP-16 in CCRF-CEM cells, produced multiple MTOCs (31).

Acetylation is possibly the primary post-translational modifier of tubulin in HL-60 cells. In undifferentiated cells, a fine rim of acetylated $\alpha$-tubulin was noted around the nucleus (Fig. 7A). In differentiated cells, acetylated $\alpha$-tubulin was most apparent as a regular radiating network of microtubules, originating from a putative MTOC in the perinuclear area, closely resembling the non-acetylated tubulin network, observed as a radiating pattern of microtubules originating from a centrosome (Fig. 7B). Dynamic microtubules grow rapidly using free unmodified tubulin, and subsequently often shrink, never existing long enough to be detectably modified. Stable microtubules simultaneously accumulate significant concentrations of post-translationally modified tubulin forms (both acetylated and tyrosinated) (32). Selective stabilization of microtubules has been discussed as a general model for morphogenesis, and acetylation and tyrosination of tubulin is shown to be a major event in differentiation-related phenomena 
A

Set-1
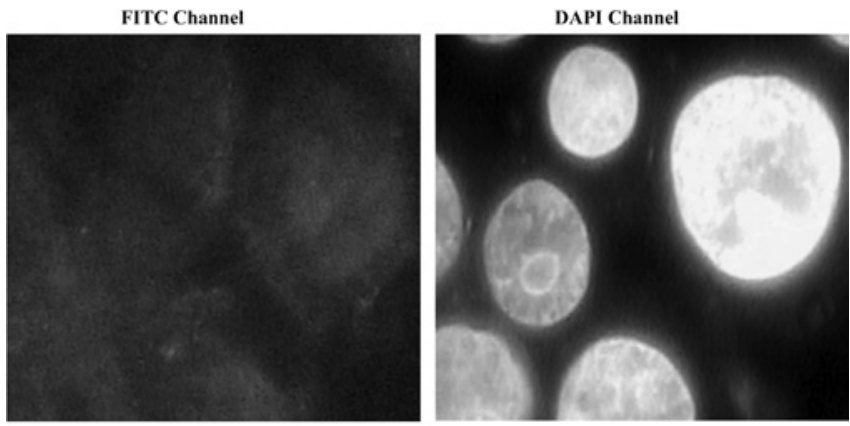

Set-2

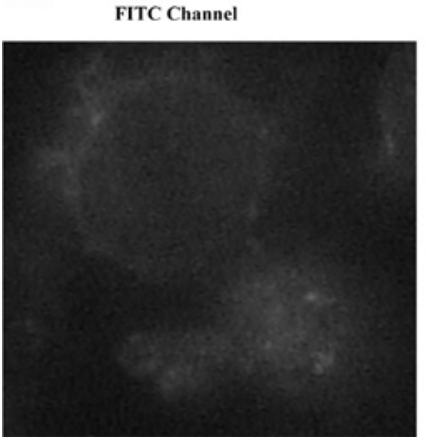

B

Set-1

FITC Channel
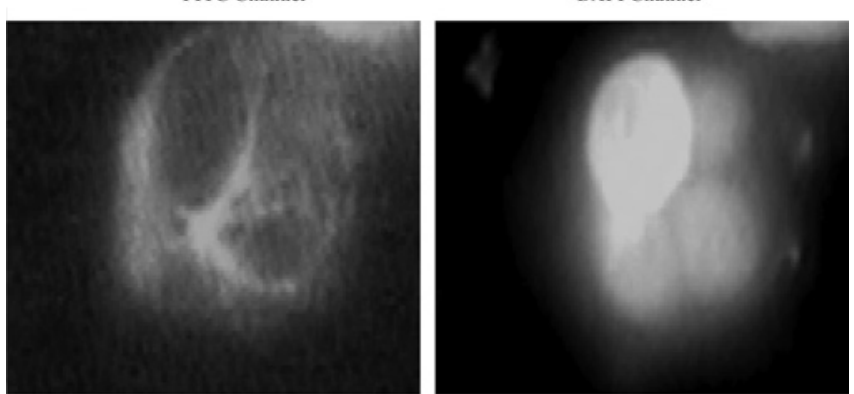

Set-2

FITC Channel
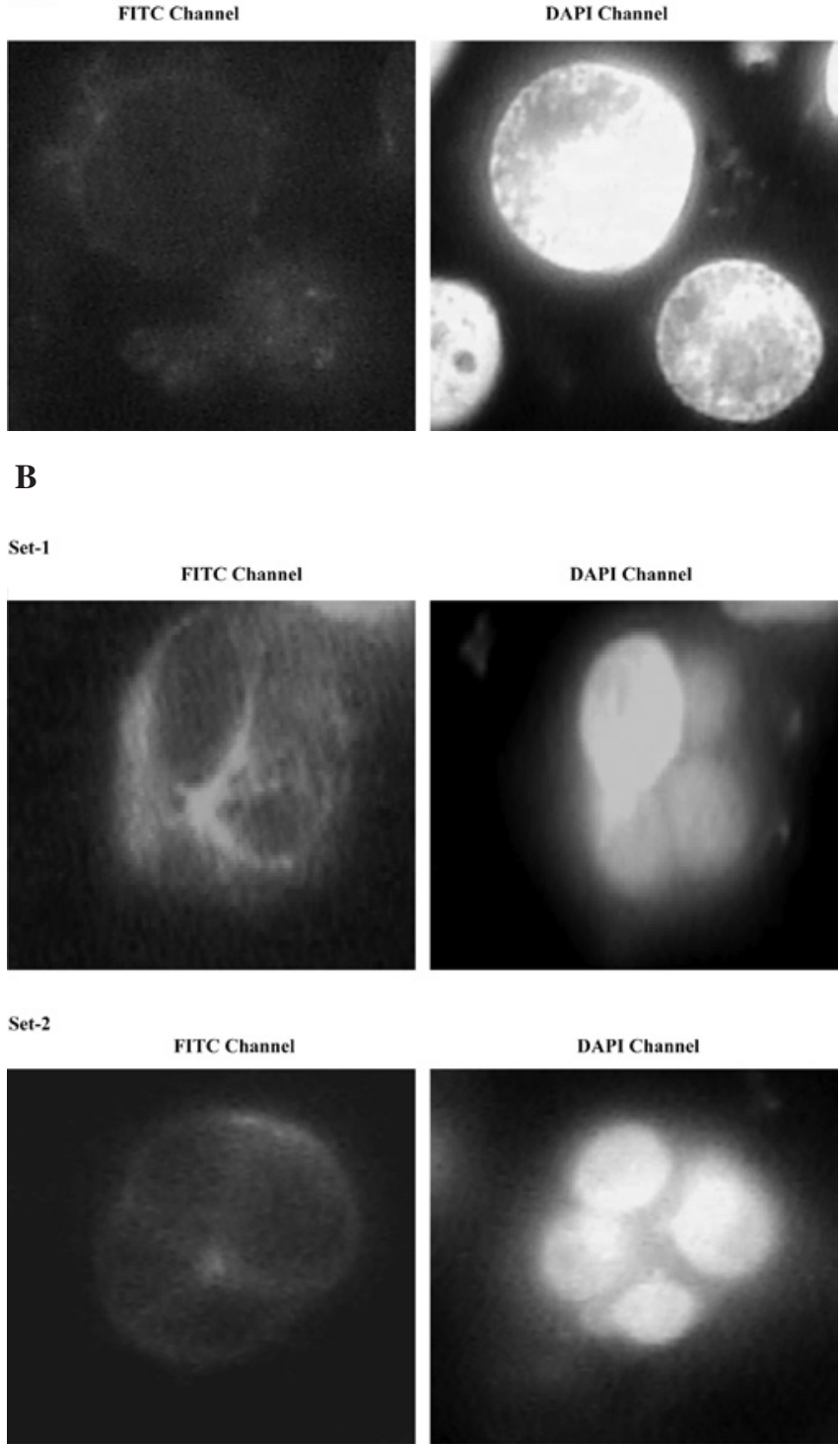

Figure 6. Nocodazole assay. Microtubules were not re-formed after nocodazole treatment (followed by a 2-h incubation with nocodazole-free media) in undifferentiated cells [(A) set-1 and -2, FITC channel]. The microtubule network is completely recovered when re-growth was allowed in nocodazole-free medium for $2 \mathrm{~h}$ after a 4-day differentiation induction by ATRA [(B) set- 1 and -2, FITC channel). Nuclear morphology was determined by Hoechst staining in the same cells [(A and B) DAPI channel].

(7). Tyrosination of microtubules was previously shown to be a key event in monocytic, but not granulocytic, differentia-

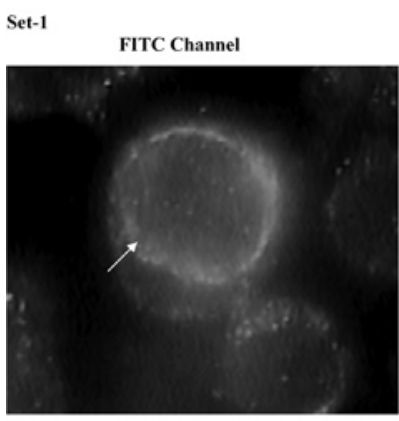

FITC Channel

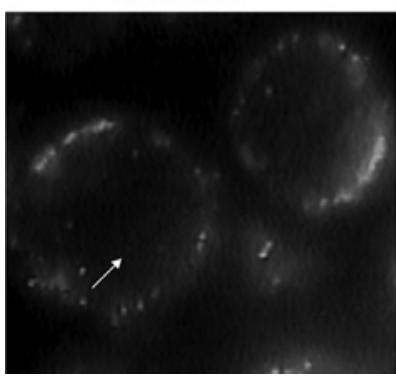

$\mathbf{B}$

Set-1

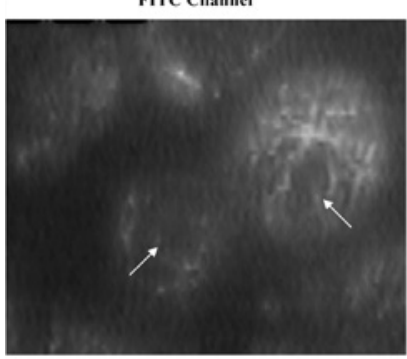

Set-2

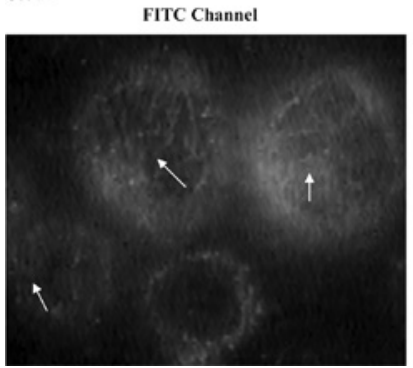

A

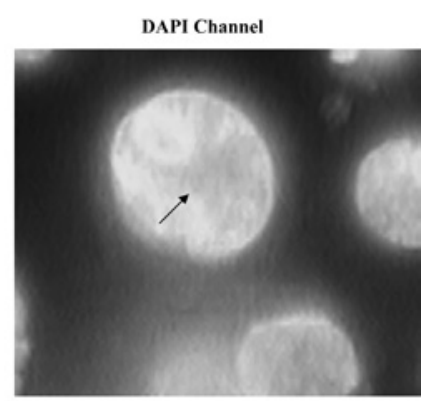

DAPI Channel
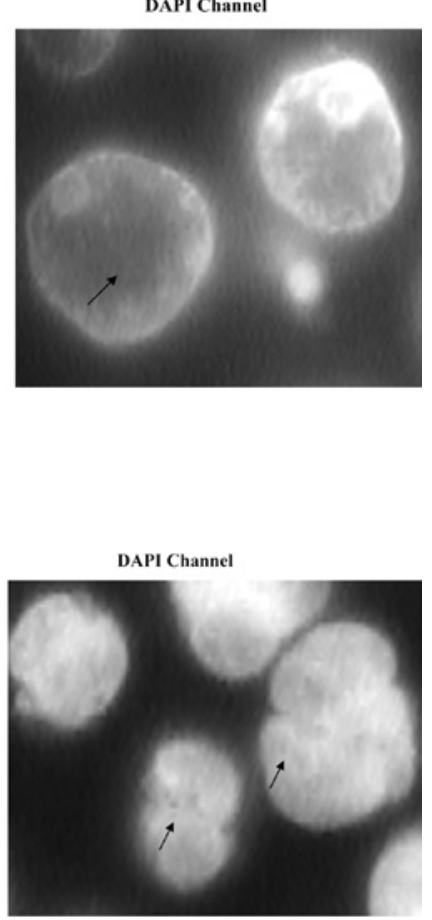

DAPI Channel

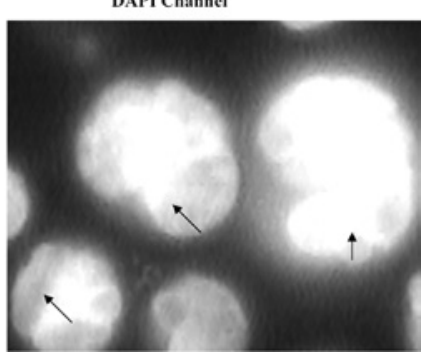

Figure 7. Immunofluorescence staining for acetylated- $\alpha$-tubulin. In undifferentiated cells, a fine rim of acetylated $\alpha$-tubulin was noted around the nucleus [(A) set-1 and -2, FITC channel]. Acetylated- $\alpha$-tubulin morphology in differentiated cells showed a radiating pattern originating from the (assumptive) centrosome [(B) set-1 and -2, FITC channel]. Nuclear morphology which distinguished between untreated, differentiated and apoptotic cells was determined in the same subset of cells using Hoechst staining [(A and B), DAPI channel].

tion of HL-60 cells (33). Olins et al found no changes in the pattern of staining for tyrosinated- $\alpha$-tubulin in differentiated and non-differentiated cells in the HL-60-S4 subclone. The content of tyrosinated-tubulin also had not changed in their 
study following differentiation of HL-60 cells. They had insignificant detection of the acetylated- $\alpha$-tubulin network in both differentiated and non-differentiated HL-60-S-4 subline by immunofluorescence microscopy, although they noted similar results in the decreased acetylated- $\alpha$-tubulin content following differentiation, as observed in our study as well (Fig. 4C) (19). Acetylated microtubules are thought to enhance the structural rigidity of the cell, and also play an active role by serving as tracks supporting the movement of organelles or vesicles, thus possibly serving as transport of CD11b vesicles in HL-60 cells $(34,35)$. So it is plausible that tubulin acetylation is the major post-translational modification of tubulin occurring during the process of granulocytic differentiation of HL-60 cells.

\section{Acknowledgements}

The authors would like to thank M. Shafiezadeh for her help in preparing the manuscript. This study was supported by the Institute of Biochemistry and Biophysics and the Research Council of the University of Tehran (640/007/6/01).

\section{References}

1. Zhang JW, Wang JY, Chen SJ and Chen Z: Mechanisms of all-trans retinoic acid-induced differentiation of acute promyelocytic leukemia cells. J Biosci 25: 275-284, 2000.

2. Robertson KA, Emami B, Mueller L and Collins SJ: Multiple members of the retinoic acid receptor family are capable of mediating the granulocytic differentiation of HL-60 cells. Mol Cell Biol 12: 3743-3749, 1992.

3. Drexler HG, Quentmeier H and MacLeod RA: Leukemia cell lines: in vitro models for the study of acute promyelocytic leukemia. Leuk Res 19: 681-691, 1995.

4. Kelly RB: Microtubules, membrane traffic, and cell organization. Cell 61: 5-7, 1990.

5. Bre MH, Pepperkok R, Hill AM, Levilliers N, Ansorge W, Stelzer EH and Karsenti E: Regulation of microtubule dynamics and nucleation during polarization in MDCK II cells. J Cell Biol 111: 3013-3021, 1990.

6. Dinsmore JH and Solomon F: Inhibition of MAP2 expression affects both morphological and cell division phenotypes of neuronal differentiation. Cell 64: 817-826, 1991.

7. Gundersen GG, Khawaja S and Bulinski JC: Generation of a stable, posttranslationally modified microtubule array is an early event in myogenic differentiation. J Cell Biol 109: 2275-2288, 1989.

8. Joshi HC, Palacios MJ and McNamara L: Gamma-tubulin is a centrosomal protein required for cell cycle-dependent microtubule nucleation. Nature 356: 80-83, 1992.

9. Felix MA, Antony C, Wright M and Maro B: Centrosome assembly in vitro: role of gamma-tubulin recruitment in Xenopus sperm aster formation. J Cell Biol 124: 19-31, 1994.

10. Schiebel E: Gamma-tubulin complexes: binding to the centrosome, regulation and microtubule nucleation. Curr Opin Cell Bio 12: $113-118,2000$.

11. Oegema K, Wiese C, Martin OC, Milligan RA, Iwamatsu A, Mitchison TJ and Zheng Y: Characterization of two related Drosophila gamma-tubulin complexes that differ in their ability to nucleate microtubules. J Cell Biol 144: 721-733, 1999.

12. Khodjakov A and Rieder CL: The sudden recruitment of gammatubulin to the centrosome at the onset of mitosis and its dynamic exchange throughout the cell cycle, do not require microtubules. J Cell Biol 146: 585-596, 1999.

13. Moudjou M, Bordes N, Paintrand M and Bornens M: Gammatubulin in mammalian cells: the centrosomal and the cytosolic forms. J Cell Sci 109: 875-887, 1996.

14. Gueth-Hallonet C, Antony C, Aghion J, Santa-Maria A, LajoieMazenc I, Wright $\mathrm{M}$ and Maro B: Gamma-tubulin is present in acentriolar MTOCs during early mouse development. J Cell Sci 105: 157-166, 1993.
15. Liu B, Marc J, Joshi HC and Palevitz BA: A gamma-tubulinrelated protein associated with the microtubule arrays of higher plants in a cell cycle-dependent manner. J Cell Sci 104: 1217-1228, 1993.

16. Kim IS, Jin JY, Lee IH and Park SJ: Auranofin induces apoptosis and when combined with retinoic acid enhances differentiation of acute promyelocytic leukaemia cells in vitro. Br J Pharmacol 142: 749-755, 2004.

17. Ozpolat B, Akar U, Steiner M, Zorrilla-Calancha I, TiradoGomez M, Colburn N, Danilenko M, Kornblau S and Lopez Berestein G: Programmed cell death - 4 tumor suppressor protein contributes to retinoic acid-induced terminal granulocytic differentiation of human myeloid leukemia cells. Mol Cancer Res 5: 95-108, 2007.

18. Brown WJ, Norwood CF, Smith RG and Snell WJ: Development of capping ability during differentiation of HL-60 human promyelocytic leukemia cells. J Cell Physiol 106: 127-136, 1981.

19. Olins AL, Herrmann H, Lichter P and Olins DE: Retinoic acid differentiation of HL-60 cells promotes cytoskeletal polarization. Exp Cell Res 254: 130-142, 2000.

20. Leung MF, Sokoloski JA and Sartorelli AC: Changes in microtubules, microtubule-associated proteins, and intermediate filaments during the differentiation of HL-60 leukemia cells. Cancer Res 52: 949-954, 1992.

21. Veselska R, Zitterbart K, Jelinkova S, Neradil J and Svoboda A: Specific cytoskeleton changes during apoptosis accompanying induced differentiation of HL-60 myeloid leukemia cells. Oncol Rep 10: 1049-1058, 2003

22. Olins AL and Olins DE: Cytoskeletal influences on nuclear shape in granulocytic HL-60 cells. BMC Cell Biol 5: 30, 2004.

23. Campbell MS, Lovell MA and Gorbsky GJ: Stability of nuclear segments in human neutrophils and evidence against a role for microfilaments or microtubules in their genesis during differentiation of HL60 myelocytes. J Leukoc Biol 58: 659-666, 1995.

24. Reibman J, Haines KA, Gude D and Weissmann G: Differences in signal transduction between $\mathrm{Fc}$ gamma receptors ( $\mathrm{Fc}$ gamma RII, Fc gamma RIII) and FMLP receptors in neutrophils. Effects of colchicine on pertussis toxin sensitivity and diacylglycerol formation. J Immunol 146: 988-996, 1991

25. Grzanka A, Grzanka D and Orlikowska M: Cytoskeletal reorganization during process of apoptosis induced by cytostatic drugs in K-562 and HL-60 leukemia cell lines. Biochem Pharmacol 6: 1611-1617, 2003.

26. VanEngelandM,Kuijpers HJ,RamaekersFC, ReutelingspergerCP and Schutte B: Plasma membrane alterations and cytoskeletal changes in apoptosis. Exp Cell Res 235: 421-430, 1997.

27. Hickstein DD, Back AL and Collins SJ: Regulation of expression of the CD11b and CD18 subunits of the neutrophil adherence receptor during human myeloid differentiation. J Biol Chem 264: 21812-21817, 1989.

28. Kiley SC and Parker PJ: Defective microtubule reorganization in phorbol ester-resistant U937 variants: reconstitution of the normal cell phenotype with nocodazole treatment. Cell Growth Differ 8: 231-242, 1997.

29. Hyman A and Karsenti E: The role of nucleation in patterning microtubule networks. J Cell Sci 111: 2077-2083, 1998.

30. Shu $\mathrm{H}$ and Joshi HC: $\gamma$-tubulin can both nucleate microtubule assembly and self-assemble into novel tubular structures in mammalian cells. J Cell Biol 130: 1137-1147, 1995.

31. Pittman S, Geyp M, Fraser M Ellem K, Peaston A and Ireland C: Multiple centrosomal microtubule organising centres and increased microtubule stability are early features of VP-16-induced apoptosis in CCRF-CEM cells. Leuk Res 21: 491-499, 1997.

32. Schulze E, Asai DJ, Bulinski JE and Kirschner M: Posttranslational modification and microtubule stability. J Cell Biol 105: 2167-2177, 1987.

33. Katagiri K, Katagiri T, Kajiyama K Yamamoto T and Yoshida T: Tyrosine-Phosphorylation of tubulin during monocytic differentiation of HL-60 cells. J Immunol 150: 585-593, 1993.

34. Piperno G, Dizet ML and Chang X: Microtubules containing acetylated $\alpha$-tubulin in mammalian cells in culture. J Cell Biol 104: 289-302, 1987.

35. Bulinski JC, Richards JE and Piperno G: Posttranslational modifications of alpha tubulin: detyrosination and acetylation differentiate populations of interphase microtubules in cultured cells. J Cell Biol 106: 1213-1220, 1988. 